\title{
Expectations of fragment decay from highly excited nuclei
}

\author{
M. Blann and M. G. Mustafa \\ Lawrence Livermore National Laboratory, P.O. Box 808, Livermore, California 94550 \\ G. Peilert, H. Stöcker, and W. Greiner \\ Institut für Theoretische Physik, Universität Frankfurt, Frankfurt am Main, Germany
}

(Received 18 March 1991)

\begin{abstract}
The statistical model is used to illustrate the consequences of a successive binary decay mechanism as the initial nuclear excitation is pushed towards the limits of stability. The partition of the excitation energy between light and heavy fragments is explicitly calculated, as are the consequences of the decay of the primary light fragments to particle-bound residual nuclei which would be observed experimentally. The test nucleus ${ }_{44}^{100} \mathrm{Ru}$ is considered at initial excitations of $100,200,400$, and $800 \mathrm{MeV}$. Exit channels of $n, p$, and $\alpha$ and 100 clusters of $3 \leq Z \leq 20,6 \leq A \leq 48$ are considered from all nuclides in the deexcitation cascade. The total primary and final cluster yields are shown versus $Z$ and initial excitation. The primary versus final yields are also shown individually for ${ }^{12} \mathrm{C},{ }^{26} \mathrm{Mg}$, and ${ }^{48} \mathrm{Ca}$. We show how multifragmentation yields will change with the excitation energy due to a successive binary decay mechanism. Measurements that may be prone to misinterpretation are discussed, as are those that should be representative of initial nucleus excitation.
\end{abstract}

\section{INTRODUCTION}

One goal in heavy-ion physics has been the investigation of the properties of nuclear matter as it is taken to the extremes of excitation. Production of highly excited nuclei may qualitatively be divided into a dynamically determined fast process in which many nucleons and some clusters escape the equilibrating mix, and a statistical part governing the properties of the equilibrated residue. The dynamic part was first predicted fairly quantitatively some years before its experimental observation [1], and several models are now available which adequately describe a broad range of the dynamic or precompound data [1,2], even to such details as pion and photon production during the thermalization process [3].

Less attention has been paid to the statistical part of the process; if an equilibrated nucleus is produced at some excitation, what are its predicted decay modes, and what should one observe experimentally to deduce information on the excitation of the parents? It is this aspect of nuclear modeling which we wish to address in the present work. In particular, we will consider heavy fragment emission properties and implications for multifragmentation in the case of successive binary emission. We consider not only primary yields, but also final yield patterns following the decay of the heavy ejectiles, which are themselves emitted with an internal energy distribution. We investigate the decay of the sample nucleus ${ }_{44}^{100} \mathrm{Ru}$ at excitations of $100,200,400$, and $800 \mathrm{MeV}$. The decay includes neutrons, protons, alpha particles, and 100 clusters of mass $6 \leq A \leq 48$ and charge $3 \leq Z \leq 20$. In this work we use a standard Fermi-gas formula for level densities; in a later work we shall investigate modifications to predictions which would result from using level densities based on shell-model single-particle levels limited to particle-bound states. Our goal is to illustrate the expectations of cluster yields from a binary statistical decay process [4], and to elucidate which experimental observables would be indicative of properties of the initial equilibrated nucleus, and which would likely be misleading. This work is done using the evaporation formalism; we refer the reader also to work which has been done in the saddle-point approximation for successive binary decay [5], and to papers investigating simultaneous multifragmentation mechanisms [6].

\section{METHOD OF CALCULATION}

Two new codes were derived from the computer code ALICE [7]. The first (referred to hereafter as code 1) used the Weisskopf-Ewing (WE) formalism [8] to calculate the decay of compound and daughter nuclei by the emission of $n, p$, and $\alpha$ plus up to 20 heavy clusters, the identity of which were specified as input. The output included product yields, double-differential cross sections (cross section versus channel energy and cluster internal excitation), differential cross sections for ejectiles stable to further deexcitation (i.e., integration over excitation below thresholds for further decay), and single-differential spectra of cross section versus internal excitation. The decay arrays considered were arbitrarily limited to a range of 18 atomic numbers $(Z)$ and 24 neutrons $(N)$ from the respective compound nucleus $N$ and $Z$.

The second code (referred to hereafter as code 2) also used the WE formalism, and was used to follow the deexcitation to bound nuclei of the heavy clusters ejected in code 1 . Code 2 treats primary ejectiles of ${ }_{20}^{48} \mathrm{Ca}$ and below, although this arbitrary limit may be changed. The code may be used to treat the deexcitation either of a single cluster or of the entire matrix produced by code 1 . Code 
2 permits decay by the emission of $n, p, \alpha$, and clusters up to half the atomic number and half the neutron number of the decaying nuclide, up to a total of 20 clusters.

Code 1 was typically run five times at each excitation so that the emission of 100 different clusters was calculated at each compound-nucleus excitation. These spectra were normalized group by group on input into code 2 , such that their cross sections were those which would result had all 100 clusters been used simultaneously competing with the $n, p, \alpha$ channels.

It is assumed that, while $n, p, \alpha$ are emitted in their ground states, all heavier clusters may be emitted in ground or excited states, with density of states given by a Fermi-gas formula [9]

$$
\rho(U)=\frac{g 6^{1 / 4}}{12} \frac{e^{2} \sqrt{\pi^{2} g U / 6}}{(g U)^{5 / 4}},
$$

where $g=6 a / \pi^{2}=(6 A / 9) / \pi^{2}$ for clusters of $A \geq 60$, with a linear interpolation for surface effects extrapolating from work by Toké and Swiatecki [10], for nuclei below $A=60$,

$$
g=\frac{6}{\pi^{2}} \frac{A}{9-2[(60-A) / 60]} .
$$

This is an approximation which we use for a first pass understanding of the expectations of the model. It would be desirable for the cluster ejectiles to use level densities based on experimental nuclear structure information where possible. This is a large project in itself which we postpone to a later time. Level densities should also be corrected by restriction to particle-bound levels. In reference to this point one should consult recent work of Hahn and Stöcker [6], Fai and Randrup [6], Grimes [11], and of Ignatyuk and collaborators [12].

Emission of $n, p, \alpha$ was calculated in the usual WE formalism as in the ALICE code,

$$
P_{v}(\varepsilon) d \varepsilon \propto\left(2 s_{v}+1\right) \mu_{v} \varepsilon \sigma_{v}(\varepsilon) \frac{\rho(U)}{\rho(E)} d \varepsilon,
$$

where $s_{v}, \mu_{v}$, and $\sigma_{v}(\varepsilon)$ are the ground-state spin, reduced mass, and inverse (reaction) cross sections, respectively. The optical model was used to calculate inverse reaction cross sections [4]. Additional details may be found in descriptions of the ALICE code [7].

For cluster decay ( $A>5$ ) we calculate

$$
\begin{aligned}
P_{v}\left(\varepsilon, E^{*}\right) d \varepsilon d E^{*}= & \mu_{v} \varepsilon \rho_{v}\left(E^{*}\right) \frac{\rho\left(E-B_{v}-E^{*}-\varepsilon\right)}{\rho(E)} \\
& \times \sigma_{v}(\varepsilon) d \varepsilon d E^{*}
\end{aligned}
$$

so that cluster spectra are calculated and stored according to the residual cluster excitation $E^{*}$ and channel energy $\varepsilon$. The intrinsic spin of the clusters does not appear as a multiplicative degeneracy factor in Eq. (4) since the total level density expression used contains a weighting due to integration over angular momenta [9]. The calculations are done deterministically using energy mesh sizes of one to three $\mathrm{MeV}$.

Code 2, which is used to treat deexcitation of the primary clusters from code 1 , uses precisely the same phys- ics. That code version uses the spectra of all (or any one) clusters versus internal excitation from code 1 as input, allowing all clusters to deexcite to particle-bound nuclei, as would be detected in an experiment. An interesting

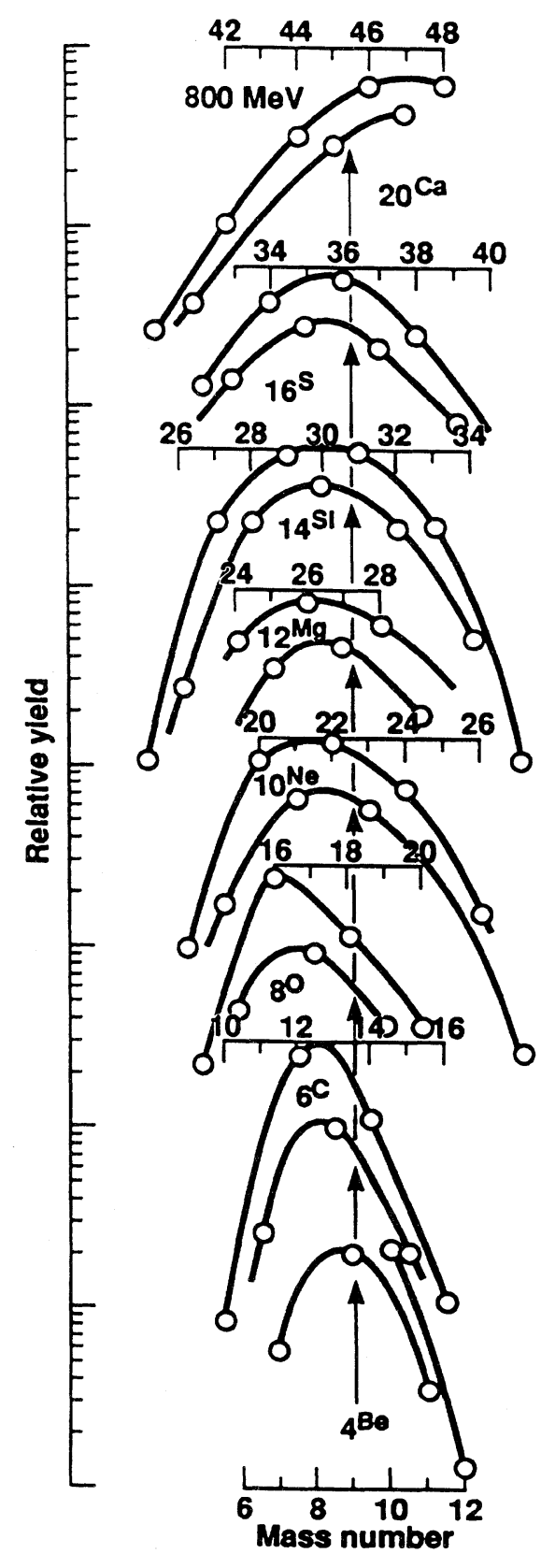

FIG. 1. Primary isotopic yields for several elements from $Z=44, A=100$ initially excited to $800 \mathrm{MeV}$ and from the daughter products of this decay. Smooth lines have been drawn through the calculated yields to illustrate the odd-even effect in the evaporation calculation. Yields for different elements have been arbitrarily displaced; the ordinate gives only correct relative yields for isotopes of a given element. Arrows indicate the position of the $N / Z$ ratio of the original compound nucleus. 
question is whether the deexcitation of the primary fragments is a minor part or a major part of their cross section. This might be rephrased as the question: Is the spectrum of, e.g., ${ }^{6} \mathrm{Li}$ measured expected to be characteristic of the Coulomb barrier and temperature of the relevant compound nucleus, or does it in fact result from the prior decay of a primary fragment such as ${ }_{10} \mathrm{Ne}$ or ${ }_{20} \mathrm{Ca}$, etc. We address these and other questions in the next section.

\section{RESULTS AND DISCUSSION}

In Fig. 1 we show relative primary yields for eight elements between $\mathrm{Be}$ and $\mathrm{Ca}$, from decay of a ${ }_{44}^{104} \mathrm{Ru}$ nucleus initially at $800 \mathrm{MeV}$ and from the daughter products of the decay chain. Results of calculation such as shown in Fig. 1 were obtained for isotopes of all ejectiles between ${ }_{3}^{6} \mathrm{Li}$ and ${ }_{20}^{48} \mathrm{Ca}$. From these the 100 isotopes having the largest emission cross sections for each atomic number were selected, so that all elements were represented with no more than $10 \%$ error due to isotopes not finally included in the calculation. The yields shown in Fig. 1 are for the primary yields before fragment deexcitation; they represent integrations over the double-differential spectra calculated versus channel energy and internal fragment excitation.

Two features of interest in Fig. 1 are the persistence of the odd-even effect at the very high excitation involved, the importance of the $Q$ values in favoring peak yields with $N / Z$ ratios different than those of the compound nuclei (indicated by arrows in Fig. 1) and the related influence of shell effects in perturbing the yield patterns for calcium and oxygen. Use of level densities which were corrected for shell structure would reduce the latter anomaly.

In Fig. 2 we show similar results for yields of isotopes of $\mathrm{Ne}, \mathrm{Mg}, \mathrm{S}$, and $\mathrm{Ca}$ from compound nuclei initially excited to 200, 400, and $800 \mathrm{MeV}$. The odd-even effect is notably enhanced at the $200 \mathrm{MeV}$ excitation.

In Figs. 3-6 we show the predicted primary distributions of fragments emitted from compound nucleus and cascade daughters for ${ }_{44}^{100} \mathrm{Ru}$ excited to $100,200,400$, and $800 \mathrm{MeV}$. The solid histograms represent the calculated primary yields, summed over all isotopes treated for each ejectile atomic number. The dotted lines represent the final yields after fragments have deexcited by $n, p, \alpha$, and cluster evaporation.

At 100 to $400 \mathrm{MeV}$ the primary and final distributions are rather similar; this is only true up to $Z=14$ at 400 $\mathrm{MeV}$, but probably would be true for all $Z$ if $Z>20$ had been included in the calculation. We will discuss the reasons for this shortly. At $100 \mathrm{MeV}$ yields for $Z>6$ drop by more than two orders of magnitude, and the fractional yields for $Z \leq 6$ are of the order of $10^{-4}$ of the reaction cross section. As the excitation goes to 200 $\mathrm{MeV}$, there is nearly a factor-of-50 increase in light fragment emission, and fragments of $Z>6$ are now down by around a factor of 20 from the lower $Z$ yields. The total fragment yields still are only a few percent of the reaction cross section. The situation has completely changed for the excitation of $400 \mathrm{MeV}$ (and above). Here all atomic number ejectiles are predicted to have similar yields (i.e., within a factor of 2-4) and heavy fragment ( $A \geq 6)$ emission has become a dominant decay mode, rather than a

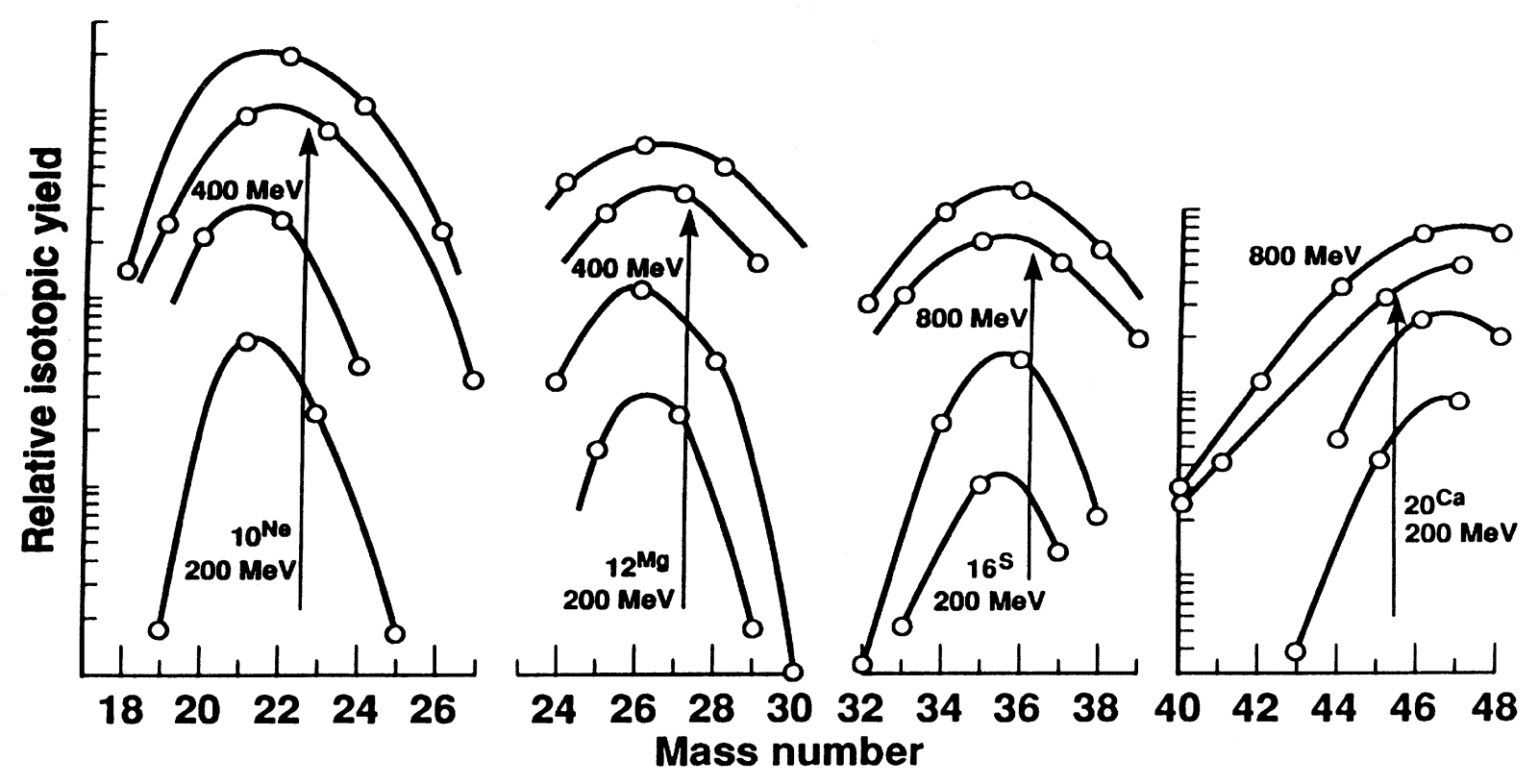

FIG. 2. As in Fig. 1, comparing relative yields of ${ }_{10} \mathrm{Ne}$ and ${ }_{12} \mathrm{Mg}$ from compound nuclei at 200 and $400 \mathrm{MeV}$ of excitation, and ${ }_{16} \mathrm{~S}$ and ${ }_{20} \mathrm{Ca}$ at 200 and $800 \mathrm{MeV}$ of excitation. 


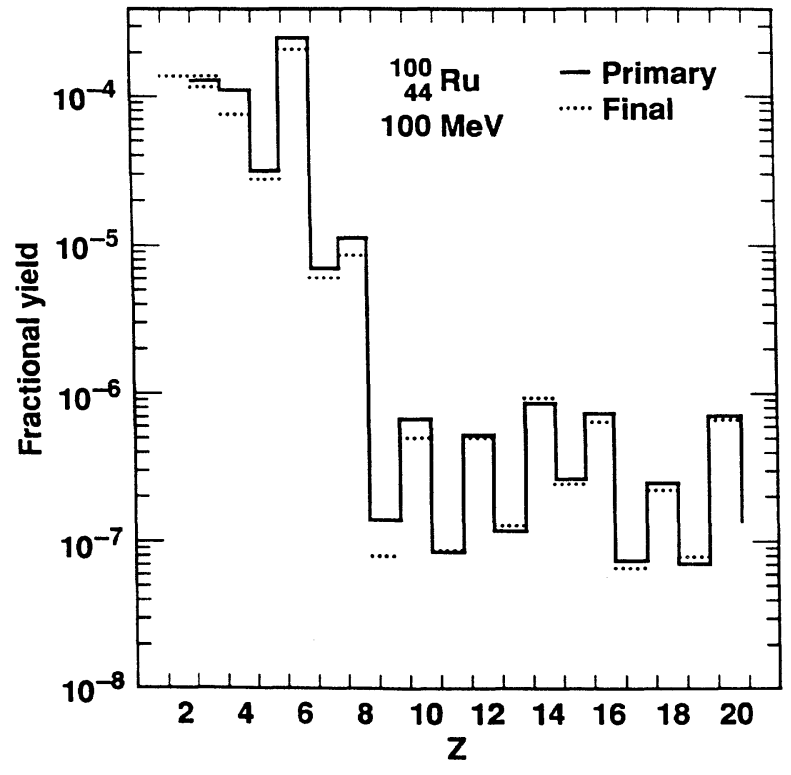

FIG. 3. Primary and final yield distributions (by atomic number $Z$ ) for the deexcitation of $Z=44, A=100$ nuclei (and cascade daughters) at an initial excitation of $100 \mathrm{MeV} .100$ light fragments from ${ }^{6} \mathrm{Li}$ to ${ }^{48} \mathrm{Ca}$ were included in the calculation, which is described in the text. The primary yields, expressed as a fraction of the compound-nucleus population, are shown by solid lines. Yields for stable nuclei, following the statistical deexcitation of the excited primary fragments, are shown by dotted lines.

channel containing but a few percent or less of the total. This situation is summarized in Fig. 7 where we show the sum of primary fragment emission $(Z \geq 3)$ versus initial compound nucleus excitation. The dashed line gives the

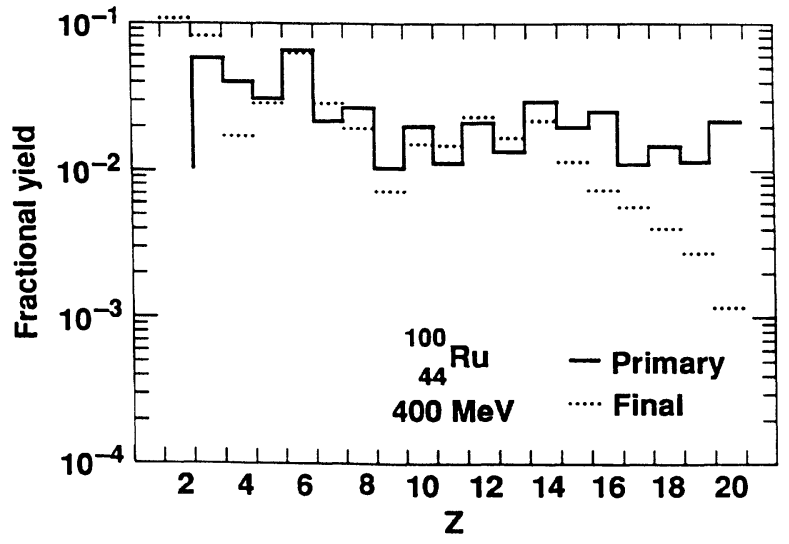

FIG. 5. As in Fig. 3 for an initial excitation of $400 \mathrm{MeV}$.

percent emission from the compound nucleus only, while the solid line gives the result averaged over the array of emitting nuclei which we have allowed to span nine atomic numbers and 18 neutron numbers for each atomic number, i.e., 162 emitting nuclei.

From Fig. 7 we may immediately make qualitative conclusions regarding fragment and multifragment decay due to an equilibrium mechanism. At excitation energies below $200 \mathrm{MeV}$ the chance of multifragmentation is very low, and rapidly decreasing with decreasing excitation; indeed the probabilities could be estimated by reading Fig. 7 for the successively lower excitations following each fragment emission process. Such an exercise shows that a very rapid increase in multifragment decay is expected at excitations above $300-400 \mathrm{MeV}$; under conditions of an equilibrated system at high excitations, multifragmentation is not a surprising experimental

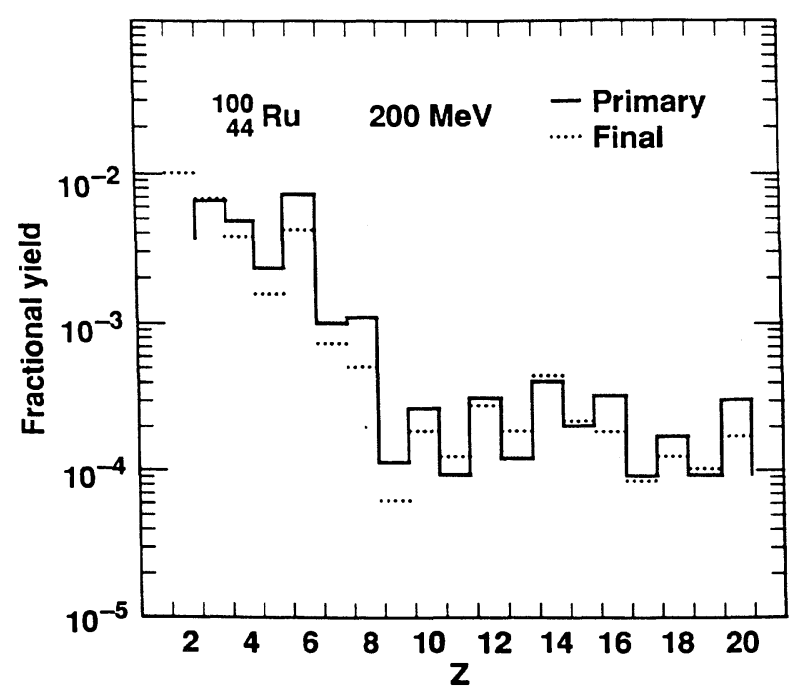

FIG. 4. As in Fig. 3 for an initial excitation of $200 \mathrm{MeV}$.

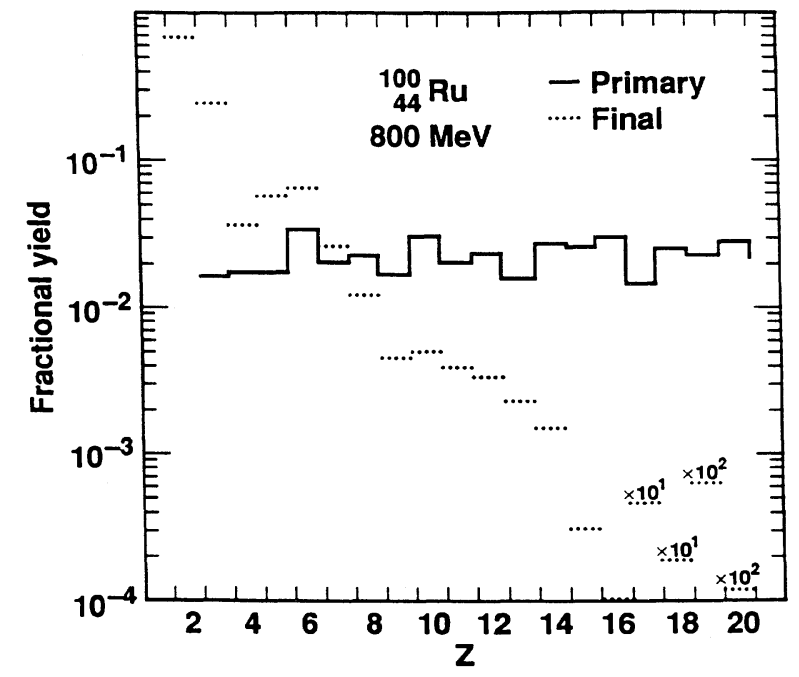

FIG. 6. As in Fig. 3 for an initial excitation of $800 \mathrm{MeV}$. 
consequence-rather it would be surprising if such a result were not found.

We wish now to interpret Figs. 3-6 more thoroughly. In order to do this we compute the final products from individual primary yield spectra of specific isotopes. We have chosen ${ }_{6}^{12} \mathrm{C},{ }_{12}^{26} \mathrm{Mg}$, and ${ }_{20}^{48} \mathrm{Ca}$ to illustrate the relevant physics. The results are presented in Figs. 8-13. In Fig. 8 it is evident that at $100 \mathrm{MeV}$, primary yields result mostly in final yields of the same atomic number. There is very little population of lower $Z$ fragments from any of the primary fragments; interpretations of, e.g., temperatures from fragment spectra and/or population of different levels (isomers) is probably reasonably valid. The similarity of primary and final yields in Fig. 3 means that there is no major charged particle deexcitation of the primary fragments; neutron and gamma decay are the principal modes of deexcitation.

In Figs. 9 and 10 there starts to be some change in interpretation at $200 \mathrm{MeV}$ of excitation. The final $\mathrm{C}$ and $\mathrm{Mg}$ isotopic yields are approximately $\frac{1}{3}$ and $\frac{1}{2}$ of the pri-

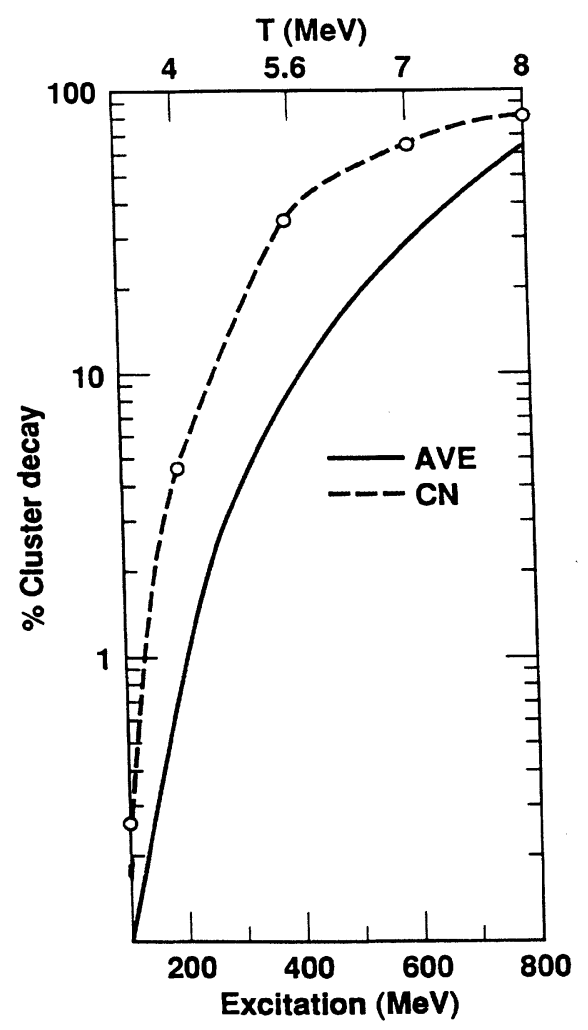

FIG. 7. Percent cluster decay (ordinate) versus initial compound-nucleus excitation energy (abscissa). The upper scale gives the nuclear temperature assuming $a=A / 8$ and $T=(E / a)^{1 / 2}$. The dashed curve which connects the open points, which are the calculated values, represents the percent of the compound nuclei decaying by cluster decay in competition with neutron, proton, and alpha particle decay. The solid curve gives the average percent cluster decay from all nuclei in a grid bounded by the compound nucleus and nuclei with 18 fewer neutrons and 8 fewer protons.
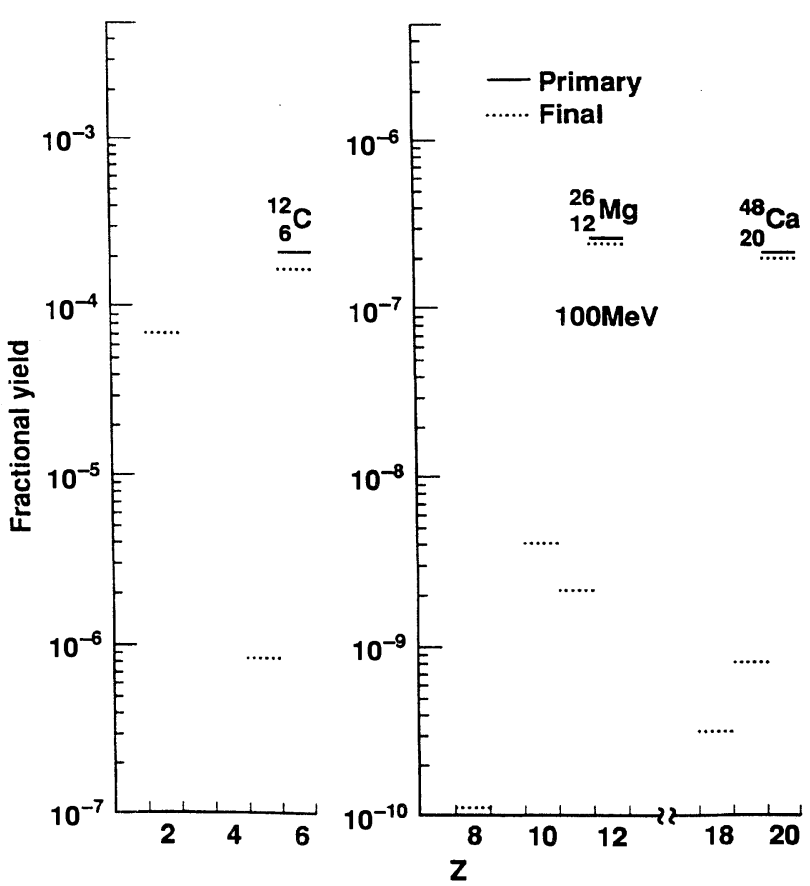

FIG. 8. The initial yield of ${ }^{12} \mathrm{C}\left({ }^{26} \mathrm{Mg},{ }^{48} \mathrm{Ca}\right)$ from decay of $Z=44, A=100$ nuclei at $100 \mathrm{MeV}$ of excitation (solid line) and the stable product yields (dotted line) following deexcitation of the excited ${ }^{12} \mathrm{C}\left({ }^{26} \mathrm{Mg},{ }^{48} \mathrm{Ca}\right)$ fragment.

mary ${ }^{12} \mathrm{C}$ and ${ }^{26} \mathrm{Mg}$ yields, whereas for $\mathrm{Ca}$ the difference is of the order of only $10 \%$ (the very neutron-rich $\mathrm{Ca}$ products decay primarily by neutron emission). The similarity of primary and final yields at $200 \mathrm{MeV}$ results

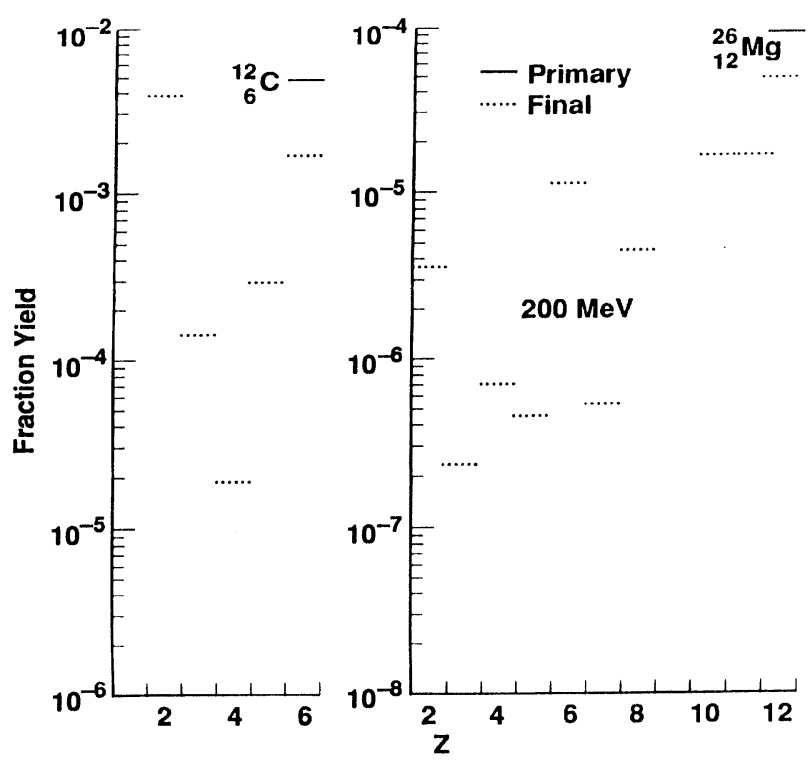

FIG. 9. As in Fig. 8 for $Z=44, A=100$ nuclei initially excited to $200 \mathrm{MeV}$ for ${ }^{12} \mathrm{C}$ and ${ }^{26} \mathrm{Mg}$ primary fragments. 


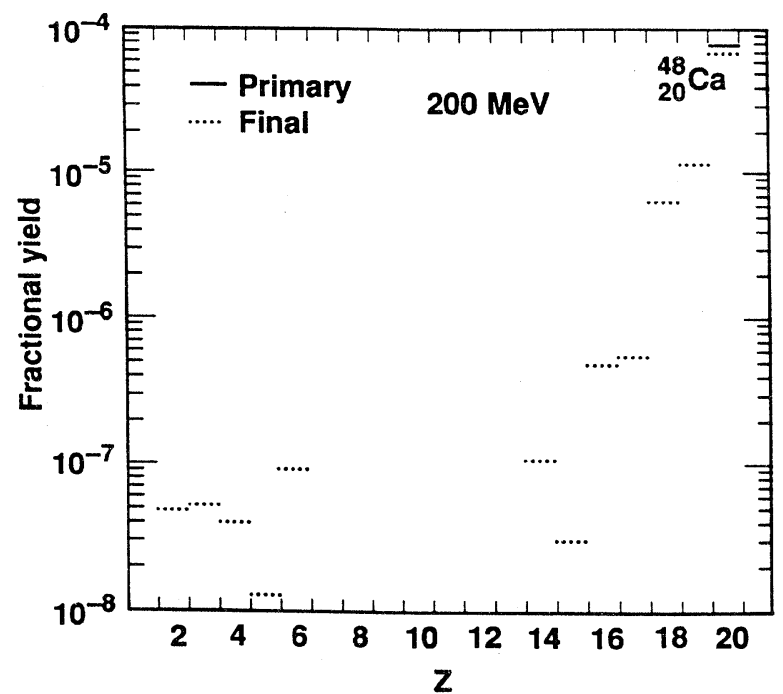

FIG. 10. As in Fig. 9 for ${ }^{48}$ Ca primary fragments.

partly because there is not a great amount of decay of primary fragments, and partly because what is lost from one primary yield is partially compensated by decay from higher mass and/or atomic number ejectiles. Under these circumstances one must begin to exercise care when interpreting spectra and/or yield patterns of ejectiles in terms of, e.g., temperature of the emitting system.

Figures 11 and 12 show the final yield patterns of the sample isotopes following decay of nuclei at $400 \mathrm{MeV}$. Here only $10 \%$ of each isotope population survives deexcitation maintaining the same atomic number (still less will maintain the same mass number). Final yields are distributed quite broadly to lower $Z$ for all isotopes; the similarity of primary and final yield patterns in Fig. 5, for $Z \leq 14$, may be seen to result from replacement of lost primary yield at each $Z$ by secondary yields from the

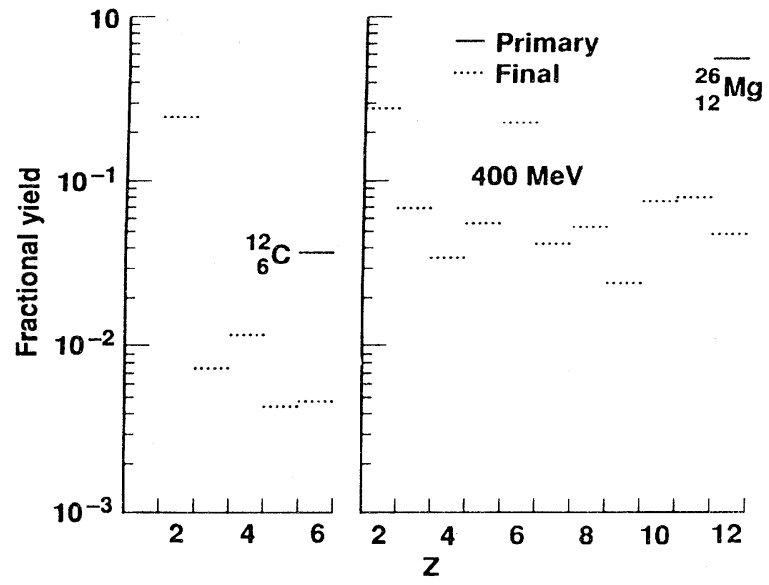

FIG. 11. As in Fig. 9 for $Z=44, A=100$ nuclei initially excited to $400 \mathrm{MeV}$.

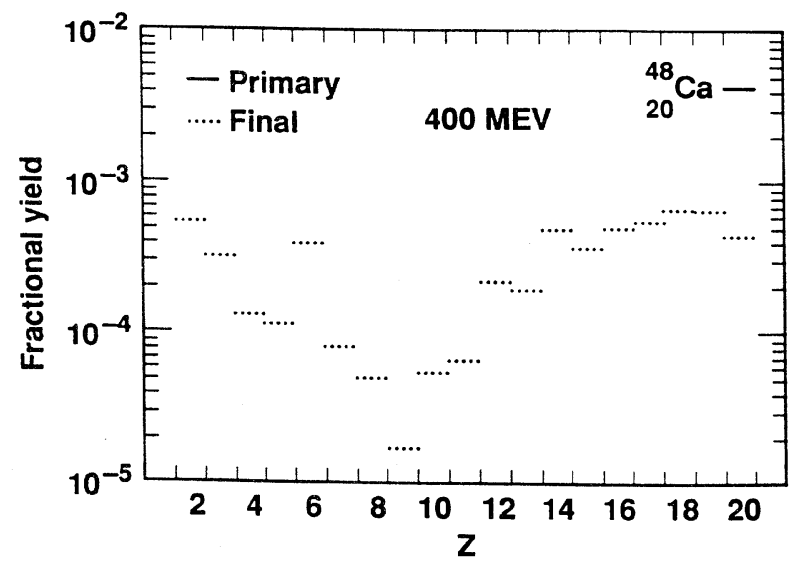

FIG. 12. As in Fig. 11 for ${ }^{48} \mathrm{Ca}$ primary fragments.

deexcitation of higher $Z$. This is the reason for our earlier statement that final and primary yields might also be similar beyond $Z=14$ had we included $Z>20$ in calculation of the primary yield spectrum.

Clearly, any attempt to interpret spectra or isomer yields of cluster ejectiles in terms of the temperature, atomic number, or other characteristics of the original nucleus is extremely dangerous. One must proceed with extreme caution.

In Figs. 6, 13, and 14 the situation may be seen to be more severe at $800 \mathrm{MeV}$ excitation energy. The primary formation yields of ${ }^{12} \mathrm{C},{ }^{26} \mathrm{Mg}$, and ${ }^{48} \mathrm{Ca}$ survive only to the order $10^{-2}, 10^{-4}$, and $10^{-4}$, respectively. Final yields are peaked toward proton and ${ }^{4} \mathrm{He}$ production with an exponential decrease as yields go toward the atomic num-

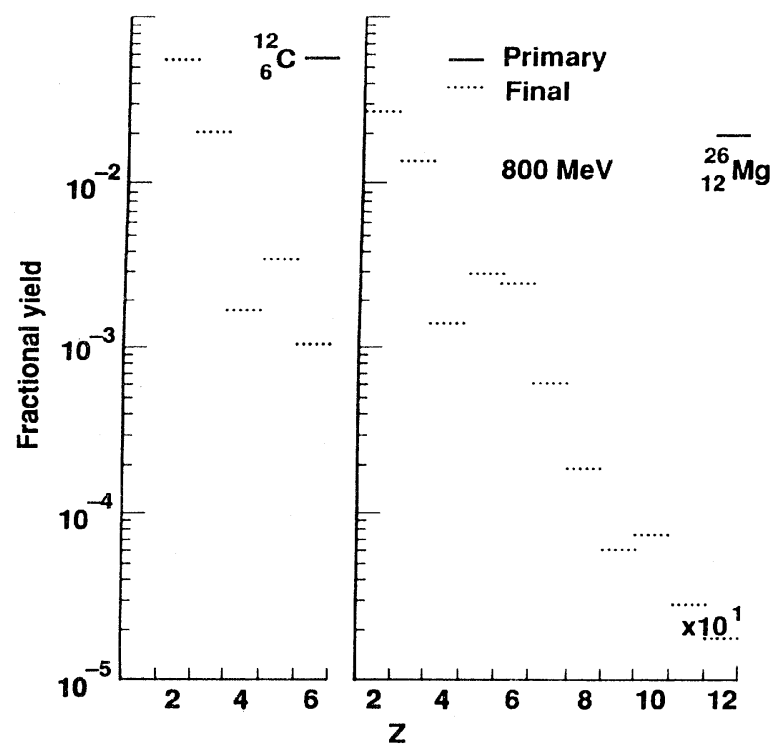

FIG. 13. As in Fig. 9 for $Z=44, A=100$ nuclei initially excited to $800 \mathrm{MeV}$. 


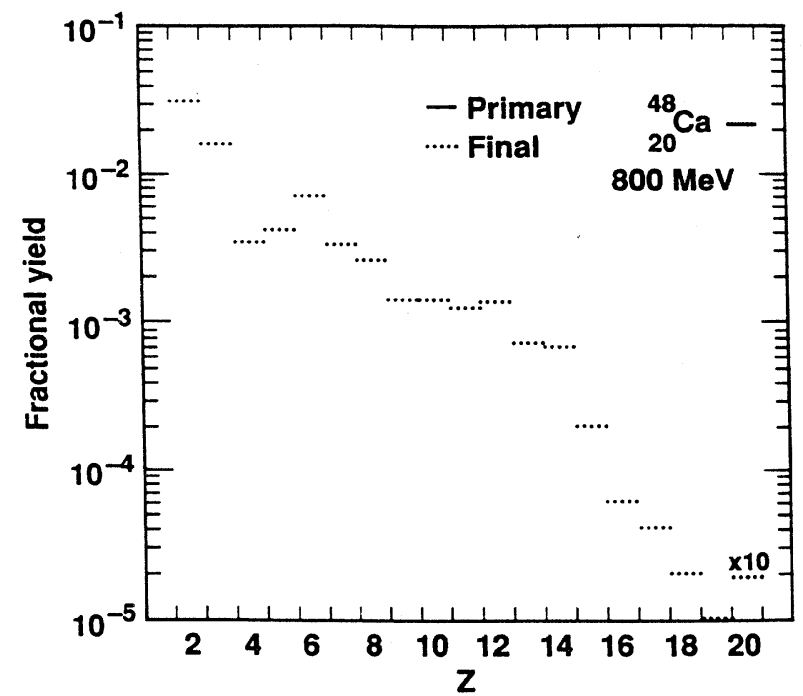

FIG. 14. As in Fig. 13 for ${ }^{48}$ Ca primary fragments.

ber of the primary fragment. The total yields of Fig. 6 would probably look more like the primary yield pattern if ejectiles of $Z>20$ had been included in the calculation; however, it appears that at such an excitation there would be a shift of final yields from heavier clusters to much enhanced yields below, e.g., $Z=14$; the enhance- ment in our limited calculation below $Z=8$ is the basis for this comment.

Figures 11-14 show that at higher initial nuclear temperatures, fragments which are observed experimentally will give very little information on the emitting nucleus excitation if, e.g., one attempts to unravel the shape of the emission spectrum or isomer yields in terms of the characteristics of the primary nucleus and daughters. This is because the emission of even a single nucleon from the relatively light fragments will considerably change their spectral distributions, and because final products will result from nuclei with temperatures considerably lower than the primary fragments. On the other hand, measurements such as the distribution of exclusive multiplicity may well allow us to deduce characteristics of the stored energy in the initial nucleus, and thereby to deduce the distribution of excited equilibrated nuclei following the initial fast cascade.

Figures 15 and 16 show contributions to the cluster decay process as a function of mass number and atomic number of the emitting nucleus. At $100 \mathrm{MeV}$ of excitation (Fig. 15) the cluster decay is $85 \%$ "first chance" emission. At $200 \mathrm{MeV}$ it is of the order of $55 \%$ first chance emission. At $400 \mathrm{MeV}$ first chance emission is of the order of $45 \%$, with contributions from a large number of cascade products. At $800 \mathrm{MeV}$ (Fig. 16) there are significant contributions from many isotopes as was the case at $400 \mathrm{MeV}$; at $800 \mathrm{MeV}$ the first chance emission continues to contribute less to the total process as the multiplicity of isotopes contributing significantly to the

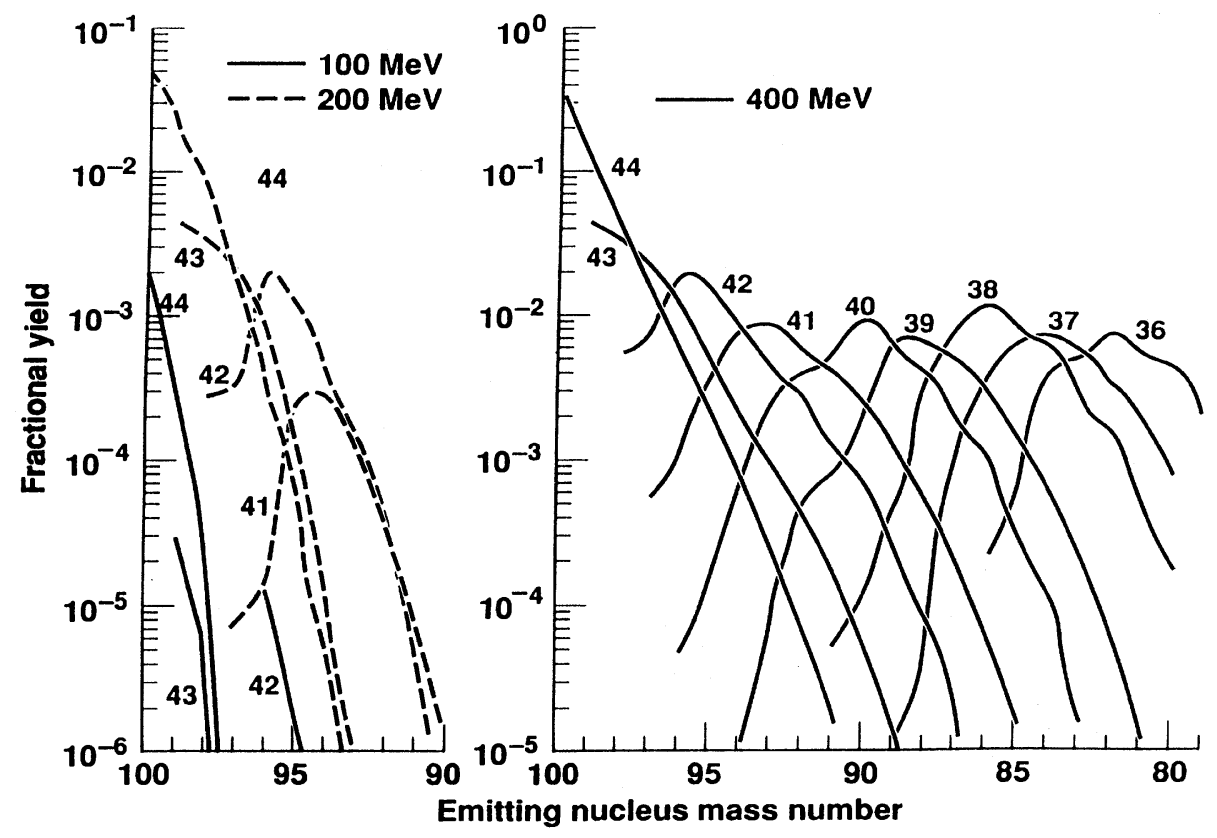

FIG. 15. Fractional cluster yield versus mass number and atomic number of the emitting nuclei from ${ }_{44}^{100} \mathrm{Ru}$ at 100 , 200, and 400 $\mathrm{MeV}$. The mass numbers are shown on the abscissas; the atomic numbers are shown above the curves which connect the yields, which are given by the intersection of the curves with each mass number. Actual calculated points have not been plotted. 


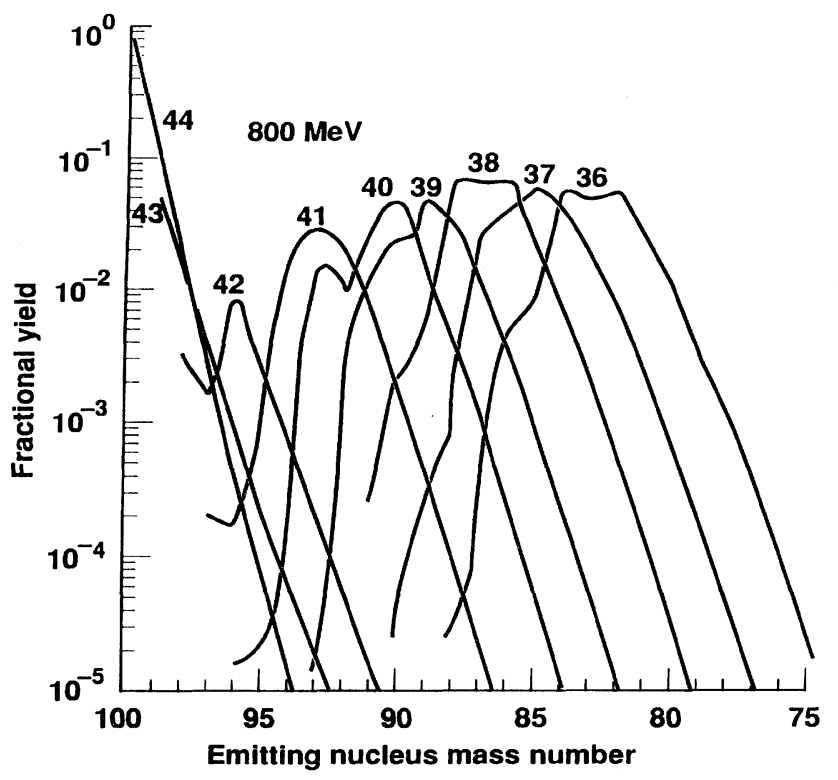
$\mathrm{MeV}$.

FIG. 16. As in Fig. 15 for nuclei originally excited to 800

decay process increases markedly.

Earlier in this work we alluded to questions concerning the validity of the Fermi-gas level density in calculations at very high excitations. Ignatyuk [12] has provided calculations for ${ }_{40}^{90} \mathrm{Zr}$ nuclei calculated with restriction to bound (either thermodynamically or centripetally) shellmodel neutron and proton energy levels. Based on these results we believe that the Fermi-gas densities used in this work are adequate up to excitations of $400 \mathrm{MeV}$, becoming progressively more questionable at higher excitations. The results at $800 \mathrm{MeV}$ should at this time be accepted with caution. Calculations of yields are probably quite reasonable because level densities appear in the numerator and denominator of the emission equations. However, lifetime calculations are sensitive to the absolute values of the level densities, and very large errors $\left(\approx 10^{5}\right)$ would result with lifetimes too short from the Fermi-gas formula. We are investigating the computational practicality of incorporating level densities based on bound realistic single-particle models into future calculations.

\section{CONCLUSIONS}

The understanding and interpretation of heavy-ion reactions requires the ability first to treat the dynamic early time scale as the nuclei begin to coalesce with fast (precompound) emission of unbound nucleons and clus- ters which escape the mix before undergoing further thermalizing interactions. The residues of this process are equilibrated nuclei. Models have been applied to treat the fast processes and seem to agree quite well with data, such as nucleon emission spectra, momentum transfer, and even production of photons and pions in the equilibration-cascade process $[1-4,13]$.

In this work we have investigated the phase-space expectations of decay of the equilibrated nuclei by successive binary decay of neutrons, protons, alpha particles, and up to 100 clusters of $A \geq 6, Z \geq 3, A \leq 48$, and $Z \leq 20$, from an arbitrary nucleus ${ }_{44}^{100} \mathrm{Ru}$, and of the successive binary decay of the clusters. We have selected the excitations $100,200,400$, and $800 \mathrm{MeV}$ to illustrate the transition from excitations where one expects to see a cluster emitted rarely, and then only one, which is probably from the compound nucleus, to the situation where multiple cluster decay is a major mode of deexcitation.

We have shown that only at the lowest excitation considered should the cluster spectra and/or isomer yields be characteristic of the compound nucleus. At higher excitations the clusters are emitted from a broad range of daughter nuclides, and then result mainly from the deexcitation of many heavier ejectiles in decay chains of varying lengths. Kinetic-energy spectra will therefore be unrelated to the primary emission spectrum of the same element; isomer ratios will correspond to the lower temperatures of fragment precursors, not to those of the heavy parents from whence the clusters were initially born.

How may we deduce the range of excitations in the original relaxed nuclei from experimental measurement? One indication would be the exclusive multiplicity distribution which is sensitive to the total stored energy. Another might be the mass yields of final products. However, each of these must be closely tied to a careful nuclear modeling effort for interpretation. Validation of the statistical models needs to be done by comparisons with relevant data at lower energies before extrapolating to higher energies. Parameters such as for the optical model for inverse reaction cross sections will be most sensitive at the lower excitations. If we cannot reproduce experimental results, e.g., for the $A=100$ region of this work at excitations of 100-200 MeV, then we should not apply the approach at higher excitations. On the other hand, if good agreement is found, we can reasonably predict results as a function of excitation, compare with models for simultaneous (rather than successive binary) deexcitation [6], and with data in order to interpret characteristics of nuclear deexcitation at very high excitation.

This work was performed under the auspices of the U.S. Department of Energy by the Lawrence Livermore National Laboratory under Contract No. W-7405-Eng48.
[1] M. Blann, in Proceedings of the International School of Nuclear Physics, Predeal Romania, 1974, edited by A. Ciocanel, (State Committee for Nuclear Energy, Bucharest, 1976), pp. 249-314; M. Blann, A. Mignerey, and W. Scobel, Nukleonika 21, 335 (1976); M. Blann, Phys. Rev. C 23, 205 (1981); Nucl. Phys. A235, 211 (1974).

[2] G. F. Bertsch et al., Phys. Rev. C 29, 675 (1984); J. Aichelin and H. Stöcker, Phys. Lett. B 176, 14 (1986); A. 
Rosenhauer et al., J. Phys. (Paris) C 4, 395 (1986); J. Aichelin et al., Phys. Rev. Lett. 58, 1926 (1987); G. Peilert et al., Mod. Phys. Lett. A 3, 459 (1988); Phys. Rev. C 39, 1402 (1989); J. Aichelin et al., ibid. 37, 2451 (1988); Y. Yariv and Z. Fränkel, ibid. 20, 2227 (1979); 24, 488 (1981); J. Cugnon, ibid. 22, 1885 (1980); J. Randrup and R. Vandenbosch, Nucl. Phys. A474, 219 (1987); A490, 418 (1988); W. Cassing, T. Biro, U. Mosel, M. Tohyama, and W. Bauer, Phys. Lett. B 181, 217 (1986); H. Stöcker and W. Greimer, Phys. Rep. 137, 277 (1986); H. Kruse, B. V. Dorak, and H. Stöcker, Phys. Rev. Lett. 54, 220 (1987); J. S. Molitoris and H. Stöcker, Phys. Rev. C 32, 346 (1985); Phys. Lett. 162B, 47 (1985); J. S. Molitoris, H. Stöcker, and B. W. Wimer, Phys. Rev. C 36, 220 (1987); C. Gregoire, B. Remaud, F. Sebille, and L. Vinet, Nucl. Phys. A465, 312 (1987); T. J. Schlagel and V. R. Pandharpande, Phys. Rev. C 36, 192 (1987); D. H. Boal and D. N. Glosli, ibid. 38, 1870 (1988); 38, 2621 (1988).

[3] M. Blann, Phys. Rev. Lett. 54, 2215 (1985); Phys. Rev. C 32, 1231 (1985); J. Aichelin, Phys. Lett. 164B, 261 (1985); B. A. Remington, M. Blann, and G. F. Bertsch, Phys. Rev. Lett. 57, 2909 (1986); Phys. Rev. C 35, 1720 (1987); B. A. Remington and M. Blann, ibid. 36, 1387 (1987); M. Blann, in The Nuclear Equation of State, Part A, Vol. 216A of NATO Advanced Study Institute, Series B: Physics, edited by W. Greiner and H. Stöcker (Plenum, New York, 1989), p. 341; W. Cassing, Z. Phys. A 329, 487 (1988); W. Bauer et al., Phys. Rev. C 34, 2127 (1986); T. S. Biro et al., Nucl. Phys. A471, 604 (1987); J. Aichelin and C. M. Ko, Phys. Rev. C 35, 1976 (1987); R. Heuer et al., Z. Phys. A 330, 315 (1988).

[4] M. Blann, in Proceedings of the 3rd International School-Seminar on Heavy Ion Physics, Dubna, 1989, (JINR, Dubna, 1990) M. Blann, T. Komoto, and I.
Tserrvya, Phys. Rev. C 40, 2498 (1989).

[5] A. Adorno, M. Colonna, M. Di Toro, and G. Russo, in Proceedings of The Nuclear Equation of State, Part A, Vol. 216A of NATO Advanced Study Institute, Series B: Physics (Ref. 3), p. 413; A. Bonasera, M. Di Toro, and Ch. Grégoire, Nucl. Phys. A463, 653 (1987); A483, 738 (1988); A. Adorno, A. Bonasera, M. Di Toro, Ch. Grégoire, and F. Gulminelli, ibid. A488, 415c (1988).

[6] J. Randrup and S. E. Koonin, Nucl. Phys. A356, 223 (1981); A424, 123 (1987); G. Fai and J. Randrup, ibid. A381, 557 (1982); A404, 551 (1983); D. H. E. Gross, Phys. Lett. 161B, 47, (1985); D. H. E. Gross, X. Z. Zhang, and S. Y. Xu, Phys. Rev. Lett. 56, 1544 (1986); D. P. Bondorf, R. Donangelo, I. N. Mishustin, C. D. Pethick, and H. Scholz, Nucl. Phys. A443, 321 (1985); D. R. Bondorf, R. Donangelo, I. N. Mishustin, and H. Schultz, ibid. A444, 460 (1989); H. Hahn and H. Stöcker, ibid. A476, 7188 (1988).

[7] M. Blann and J. Bisplinghoff, Lawrence Livermore National Laboratory Report UCID-19614, 1982 (unpublished); M. Blann and H. K. Vonach, Phys. Rev. C 28, 1475 (1983).

[8] V. F. Weisskopf and D. H. Ewing, Phys. Rev. 57, 472 (1940).

[9] T. E. O. Ericson, Adv. Phys. 9 (36), 425 (1960).

[10] J. Toké and W. J. Swiatecki, Nucl. Phys. A372, 141 (1981).

[11] S. M. Grimes, private communication.

[12] A. V. Ignatyuk, private communication.

[13] M. Blann, Phys. Rev. C 31, 295 (1985); in Proceedings of the 17th International Summer School on Nuclear Physics, Nuclear Science Series, Trends in Nuclear Physics, edited by Z. Wilhelmi, G. Szeflinska, and M. Kicinska-Habior (Harwood Academic, London, 1985), p. 295. 